\title{
8. Theory of Tip-Sample Interactions
}

\section{S. Ciraci}

With 10 Figures

In conventional, Scanning Tunneling Microscopy (STM) the tip-sample separation is assumed to be sufficiently large to allow only weak coupling between the electronic states. In this case the electrodes have been considered to be independent. In some operating modes of STM the tip-sample separation is purposely set small to enhance the tip-sample interaction and hence to modify the electronic and atomic structure irreversibly. Indeed, as the tip approaches the sample surface, the potential barrier is lowered, the charge density is rearranged and the ions in the vicinity of the tip are displaced to attain the minimum of the total energy at the preset tip-sample distance. Modifications of the electronic and atomic structure depending upon the tip-sample separation have led to the identification of different regimes in the operation of STM; ranging from the independent electrodes to the irreversible mechanical contact. This chapter deals with the tip-sample interaction effects. The variation of electronic structure and vacuum barrier, the character of conduction and tip force are investigated as a function of separation. Our analysis suggests that operation of the tunneling and force microscopes under a significant tip-sample interaction will bring about potential applications not only in the investigation of electronic and atomic structure but also in mesoscopic physics.

\subsection{Tip-Sample Interaction}

The invention of scanning tunneling microscopy [8.1] and subsequently of scanning force microscopy (SFM) [8.2] provided new techniques for studying the structure and the electronic properties of solid surfaces and molecules down to the atomic scale. The conventional view of STM usually assumes that the tip-sample separation $z$ is large and hence the electrodes (i.e., tip and sample) are nearly independent. In this case, the tunneling can be described by the transfer Hamiltonian approach in terms of one-electron states of the bare electrodes (i.e., free tip and free sample) [8.3]. Using the transfer Hamiltonian approach and representing the tip by a single s-wave it was shown that the tunneling is approximately related to the local density of the electronic states of the sample at the center of the tip at the Fermi level, $\varrho_{\mathrm{S}}\left(\boldsymbol{r}_{0}, E_{\mathrm{F}}\right)$ [8.4]. Accordingly, the tunneling current is exponentially dependent on the tip-sample separation, and STM probes the weak overlap between the wave functions of electrodes yielding a resolution down to the atomic scale. 
Depending on the bias polarity, tunneling occurs either from filled or towards empty states of the sample and can also reveal the spectrum of the sample surface in the range of energy $E_{\mathrm{F}}-e V \leq \varepsilon_{\mathrm{S}} \leq E_{\mathrm{F}}+e V\left(E_{\mathrm{F}}\right.$ and $V$ being Fermi energy and bias voltage, respectively) $[8.5,6]$. New techniques, such as ballistic electron emission microscopy [8.7] (to probe interfaces and to provide spatial and energy resolution of the scattering process) and field emission of electrons from an atomically sharp tip [8.8] (to obtain a stable and well collimated e-beam) are also derived from STM. Even though the coupling between the electronic states is negligible, the correlated motion of the electrons in separated electrodes gives rise to a long-range interaction. The van der Waals (VdW) forces derived from this long-range interaction may be significant depending on the shape of the tip, but are expected to be essentially uncorrugated on the atomic scale. All these modes of operation and long-range interaction in STM occur at a large separation with nearly independent electrodes being identified as the conventional tunneling regime.

In some typical operating modes of STM [8.9], the observation of force variation of the order of $10^{-9} \mathrm{~N}$ (or several $\mathrm{eV} / \AA$ ) indicated significant overlap and rearrangements between electronic charge densities of sample and tip, at least if just a few atoms are responsible for these variations. In several other studies, the tip-sample separation was purposely set small to modify atomic and electronic structure of electrodes and hence to enhance the current. STM operating at small $z$ (corresponding to very small bias) yielded a corrugation which is much larger than that deduced from $\varrho_{\mathrm{S}}\left(r_{0}, E_{\mathrm{F}}\right)$, e.g. of the free graphite surface [8.10]. Initially the observed giant corrugation was attributed to the elastic deformation indicating a significant tip-sample interaction owing to the close proximity of the tunneling tip. Self-Consistent Field (SCF) pseudopotential calculations had shown, however, that as $z$ decreases the potential barrier $\Phi_{\mathrm{B}}$ gradually collapses, and the surface charge density of graphite is disturbed locally [8.11]. The electronic states, which are relevant for tunneling evolve into a kind of resonance states having relatively higher weights in the vicinity of the tip [8.12]. Tip-induced local disturbances of the electronic states may change into a chemical bond between the outermost tip and sample atoms [8.11]. STM observations of individual "atoms" with the periodicity of nominally flat (111) surfaces of close-packed noble [8.13] and simple [8.14] fcc metals with a corrugation much larger than one would deduce from $\varrho_{\mathrm{S}}\left(\boldsymbol{r}_{0}, E_{\mathrm{F}}\right)$ suggest site-dependent tip-surface interaction effects [8.15]. Whether force variations and induced deformations along STM scans and/or changes in electronic structure (together with the contribution of d-states) must be invoked to explain such observations is understandably an important issue. The interaction energy $E_{\mathrm{i}}(z)$ (or adhesion energy) between tip and sample, and the short-range force derived thereof are important outcomes of the tip-sample interaction. The tip force is of relevance for SFM when it shows significant variation with the tip position [8.16]. This occurs at relatively small $z$, in which short-range forces dominate the tip force. As $z$ is decreased, the interaction energy becomes increasingly negative until the separation $z=z_{\mathrm{e}}$ corresponding 
to maximum adhesion. The perpendicular tip force $F_{\mathrm{s} \perp}(z)=-\partial E_{\mathrm{i}}(z) / \partial z$ becomes first increasingly attractive, passes through a minimum and then decreases to become repulsive for $z<z_{\mathrm{e}}$. For an atomically sharp tip it is expected that significantly strong lateral forces can also arise when the tip is positioned off high symmetry positions. If the lateral force gradient exceeds the restoring spring constant of the cantilever, the tip starts to perform a stick-slip motion on the sample surface [8.17]. These lateral forces, which are essentially conservative, can thus induce hysteresis and losses via energy transfer to shear modes, resulting in an average microscopic friction force of nonconservative nature.

It becomes clear from the above discussion that the evolution of the electronic and transport properties and the variation of the tip force with the tip-sample separation distinguish new regimes of operation in STM, which are different from the conventional tunneling between nearly independent electrodes $[8.12,18,19]$. While the conventional tunneling has developed into a powerful technique with real-space imaging capability and atomic resolution, new regimes in the presence of significant tip-sample interaction may open new horizons in the application of STM and SFM [8.18]. As the distance between the tip and sample is decreased, the overlap of the wave functions of the electrodes increases and several interrelated atomic scale interaction effects then come into play, as suggested by investigations of the transition from tunneling to electrical and mechanical contact [8.20]. The potential barrier between tip and sample is gradually lowered, which causes significant rearrangements of the charge density. This, in turn, induces an attractive (binding) interaction or adhesion energy leading to short-range attractive forces. Responding to the latter, the ions of the tip and sample are displaced even before the irreversible (plastic) deformations set in. A few ångstrom before mechanical contact, reversible local electronic and structural modifications are expected. The potential barrier collapses before the point of maximum attraction on the apex of the tip. This regime at intermediate separations is characterized by significant electronic interaction and is identified as the electronic contact regime. In spite of the fact that the electronic states are modified, the transport of current takes place via tunneling.

Upon a further approach of the tip, a mechanical contact is eventually formed through strong bonds with sample atoms [8.11]. If the cross section of the contact is sufficiently large the constriction effect due to the confinement of current carrying states becomes negligible, hence the transport occurs in the absence of any barrier. This is the ballistic conduction and involves quantum effects since the size of the contact is comparable with the Fermi wavelength $\lambda_{\mathrm{F}}$. The operation of STM in this range of very small separation reveals a different regime, in which the character of transport can undergo a qualitative change [8.20-24]. Irreversible deformations are then also expected in the vicinity of the tip $[8.25,26]$. We identify this regime as that of mechanical contact.

In Fig. 8.1 these new regimes (i.e., electrical and mechanical contact regimes), which occur beyond that of the conventional tunneling and involve significant 


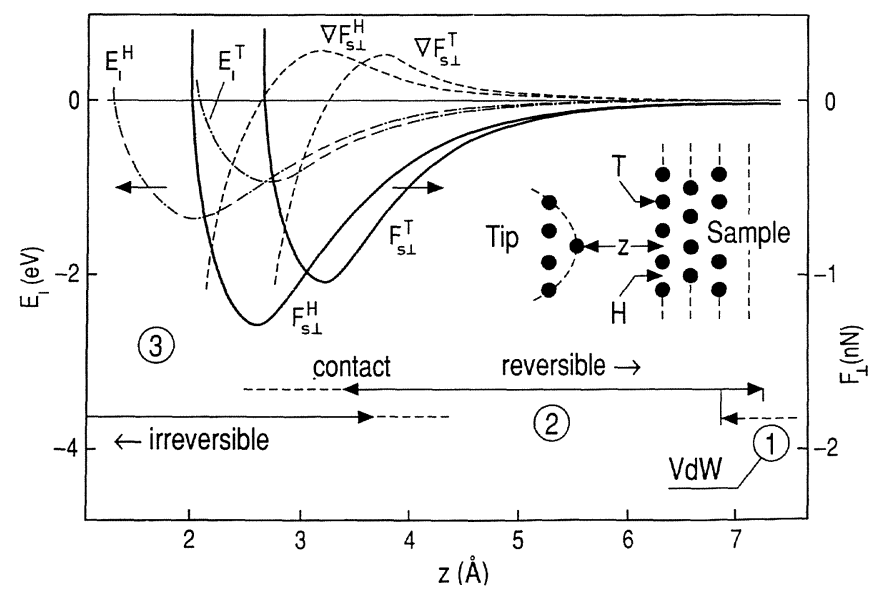

Fig. 8.1. Interaction energy $E_{\mathrm{i}}(z)$, short-range perpendicular force $F_{\mathrm{s} \perp}$ and force gradient $\nabla F_{\mathrm{s} \perp}(z)$ (in arbitrary units) calculated for the $\mathrm{Al}(001)$ sample and tip positioned at the hollow $(\mathrm{H})$ and top (T) sites described by the inset. Ranges of separation $z$ for various regimes of operation in STM are schematically indicated

tip-sample interactions, are schematically represented with reference to physical quantities such as interaction energy $E_{\mathrm{i}}(z)$, the perpendicular component of the short-range tip force $F_{\mathrm{s} \perp}$, and its gradient $\nabla F_{\mathrm{s} \perp}$ [8.27]. Of course, the extent of these regimes varies depending on the electronic and structural properties of the tip and sample. Besides, the transition between adjacent regions is not sharp.

In this chapter we present a detailed analysis of tip-sample interaction effects on the tip force and transport properties. This is achieved by examining the evolution of electronic states, the potential barrier intervening between two electrodes, and interaction energies as a function of separation. Since the theory relevant to the conventional tunneling has been treated in a number of publications $[8.4,5,28]$, we consider here only the effects of long-range interactions. The focus of the chapter is, however, the electrical and mechanical contact regimes. Since the tip force which is relevant for SFM operating at small $z$ has a short-range character, the force microscope is also included in the scope of this chapter. Based on the results of ab-initio calculations we examine the modifications of electronic states and the potential barrier leading to tip-induced localized states. The atom transfer between tip and sample attracts interest since it underlies the structural modification of the sample at the atomic scale. Here we also outline results of recent ab-initio calculations, which are relevant for the atom transfer.

Another objective of this chapter is to reveal important features of STM which pertain to mesoscopic physics. It has now become clear from recent works that the atomically stable sharp tips can be fabricated [8.8]; the cross section relevant for electron transmission is in the range of atomic dimensions or the Fermi wavelength $\lambda_{F}$. Therefore, we discuss the formalism of transport through 
a quasi one-dimensional (1D) constriction which was developed earlier [8.29]. Recently, starting from such a formalism, a method was developed to treat the transport between tip and sample surface [8.24]. Since this theory has validity in a range covering tunneling as well as ballistic conduction, physical events involving these separately or concomitantly, and the transition between them, can successfully be addressed. The effective potential barrier, which is generated due to the lateral confinement of states between tip and sample, can be easily visualized within the framework of this method, and will be shown to have important implications. Interesting features of $\log I(z)$ curve, such as saturation at the first plateau and large period "quantum" oscillations observed at the mechanical contact are also covered. Finally, we touch upon an interesting issue, namely exploring a qualitative relation between the short-range tip force and tunneling conductance to unify certain concepts in STM and SFM.

\subsection{Long-Range (van der Waals) Forces}

The origin of the van der Waals (VdW) force can be better described in terms of surface plasmons of the electrodes, which are coupled through their electric field [8.30]. Therefore, the VdW force is long ranged and can occur even if the electronic states of separated electrodes are decoupled and hence the exponentially decaying short-range forces are negligible. The importance of the $\mathrm{VdW}$ force was recognized earlier, and it was argued that, depending on the shape of the tip, the outermost atoms experience strong repulsion, even irreversible deformation, due to the body forces (or VdW forces) at the back of the apex $[8.18,19]$. The role of the VdW forces in SFM has been treated by various authors [8.9, 31-33]. Existing studies rely on the integration of the basic power law over the volume of the probing tip.

Although the continuum description of electrodes leaves out the discrete nature of the tip, the generalized Lifshitz approach [8.34] is, nevertheless, convenient for metal electrodes. In the case of flat parallel electrodes, the electric field of a plasmon mode with a wave vector $\boldsymbol{k}_{\|}$varies with $\exp \left(-k_{\|} z\right)$ and, hence, the VdW interaction is dominated by the long wavelength modes at separation $z \gtrsim 14$ a.u. [8.30]. This is the limit leading to the Lifshitz formula [8.34] which, in turn, diverges as $z \rightarrow 0$, so it cannot be adequate to represent the $\mathrm{VdW}$ interaction for small $z$. With the same reason the shape of the tip becomes crucial at small $z$. The correct description of the VdW then requires a proper account of the collective behavior of the electrons with nonlocal microscopic dielectric theory. In SFM not only the VdW force alone, but its gradient may be important [8.32]. At large $z$ (where the short-range force is negligible), the force gradient of the VdW force becomes relevant if it exceeds the force constant of the cantilever. This causes an inelastic instability in which the tip jumps to the range $z \simeq z_{\mathrm{e}}$. At small $z$, the force gradient of the short range force may be added to that of the VdW force. 
Integrating the Lifshitz formula with appropriate Hamaker constant [8.35]

$$
E_{\mathrm{W}}=-\frac{A}{6 \pi} \int_{V_{\mathrm{tip}}} \frac{\mathrm{d} \boldsymbol{r}}{[z(\boldsymbol{r})]^{3}},
$$

for semi-infinite tips (with conical and hemispherical ends and cylindrical shank) the VdW interaction energy can be calculated [8.33]. In (8.1) $z(r)$ is the height of the differential volume element located at $r$ on the tip, and $A$ is the Hamaker constant having the value $3.6 \times 10^{-19} \mathrm{~J}[8.32,35]$. By varying the cone angle $\alpha$, the diameter of the shank and $z$ independently, a wide range of tip geometry can be taken into account to investigate the long-range interaction [8.33]. The VdW force, $F_{\mathrm{W}}$ is calculated by differentiating (8.1). Results relevant for the present discussion are summarized in Fig. 8.2. These calculations have indicated that for the hemispherical geometry the VdW force and its gradient are large, and their values increase with increasing diameter. For large values of the force gradient $\left(\nabla F_{\mathrm{W}} \gg \sim 1 \mathrm{~N} / \mathrm{m}\right)$ the probing tip jumps to contact and creates instabilities. On the other hand, the VdW force is only $\sim 0.1 \mathrm{~N}$ for a conical tip having $\alpha=45^{\circ}$ and $z \simeq 14$ a.u. For the same tip and separation, the force increases by more than one order of magnitude if $\alpha$ increases to $75^{\circ}$, but it decreases by two orders of magnitude when $\alpha$ is decreased to $5^{\circ}$. The force gradient for the conical tip of $\alpha=5^{\circ}$ is also small and is in the range of $0.1 \mathrm{~N} / \mathrm{m}$. In contrast, the gradient of the short-range force in the increasingly attractive range is $\sim 1 \mathrm{~N} / \mathrm{m}$. It appears that apart from instabilities which can be avoided by using a stiff cantilever, the VdW interactions cannot have a significant effect on atomic resolution measurements with sharp tips in STM.

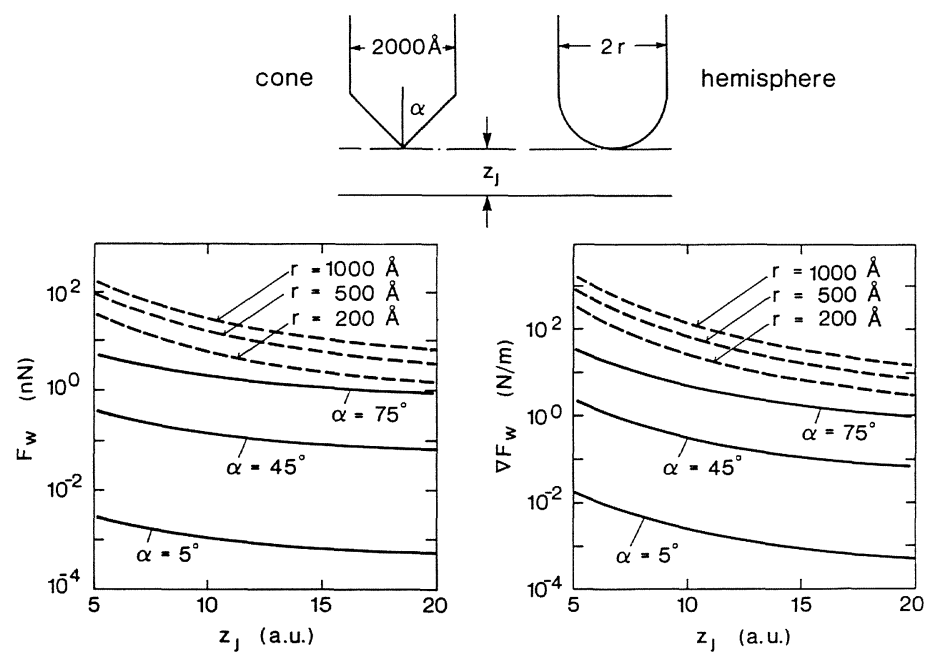

Fig. 8.2. Van der Waals force, $F_{\mathrm{w}}$ and its gradient, $\nabla F_{\mathrm{W}}$ calculated as a function of tip-sample separation $z_{j}$ (measured from their jellium edges) for different tip geometries 


\subsection{Interaction Energy: Adhesion}

When two semi-infinite slabs are close to each other the electrostatic repulsion is overcome by the rearrangement of electronic states yielding net attraction (electrostatic plus exchange-correlation energy) at the expense of positive kinetic energy. The interaction energy $E_{\mathrm{i}}(z)$ is obtained by subtracting the total energies of the tip $E_{\mathrm{t}}$ and sample $E_{\mathrm{S}}$ alone from the total energy of tip and sample together but separated by $z[8.19,33]$

$$
E_{\mathrm{i}}(z)=E_{\mathrm{t}+\mathrm{s}}(z)-E_{\mathrm{t}}-E_{\mathrm{S}} .
$$

By definition $E_{\mathrm{i}}(z)<0$ indicates an attractive (binding) interaction. The minimum of $E_{\mathrm{i}}(z)$ at $z=z_{\mathrm{e}}$ is identified as the binding energy $E_{\mathrm{b}}$ of the electrodes (in which the VdW interaction is not taken into account). It is customary to define the adhesive energy (per atom) for flat electrodes $E_{\mathrm{ad}}(z)=E_{\mathrm{i}}(z) / 2$ which is the negative of the amount of work necessary to separate two semi-infinite slabs from $z$ to $\infty$. Note that the surface energy is the negative of $E_{\mathrm{i}}\left(z=z_{\mathrm{e}}\right) / 2$ calculated for two such slabs. The binding energies between two semi-infinite slabs can be calculated by using the jellium approach [8.36], whereas, the binding energy between an atom (or group of atoms) representing the tip and sample surface can be obtained by using supercell geometry allowing the periodic boundary conditions [8.19]. Earlier, the binding energy of a single Al atom on a graphite surface at the top and hollow site were calculated by using the pseudopotential method within the Local Density Approach (LDA). The binding energies were found rather small, $E_{\mathrm{b}}=-0.33 \mathrm{eV} /$ atom for the top (T) site and $-0.61 \mathrm{eV} /$ atom for the hollow $(\mathrm{H})$ site [8.19]. On the other hand, the binding energy between two $\mathrm{Al}(001)$ slabs is significantly larger, i.e., -0.92 and $-1.37 \mathrm{eV} /$ atom for the top and hollow sites, respectively [8.33].

The interaction energy between two thin $\mathrm{Al}(001)$ slabs is presented in Fig. 8.3 for the T- and H-site [8.33]. An important observation is that even for a simple metal the site dependence is significant. $E_{\mathrm{b}}$ is larger at the $\mathrm{H}$-site since the resulting stacking corresponds to the natural one of the $\mathrm{Al}(001)$ layers in bulk Al. However, beyond the separation of maximum attraction at the T-site the $E_{\mathrm{i}}(z)$ curve is lowered below that of the $\mathrm{H}$-site. For $z \gtrsim 10$ a.u. both curves are merged into a single curve and decay exponentially. The interaction energy is short ranged since it is determined by the overlap of charge densities which decay exponentially. It is therefore reasonable to expect that for $z>z_{\mathrm{e}}, E_{\mathrm{i}}(z)$ can be approximated by an exponential function. Earlier, Rose et al. [8.37] proposed a simple universal relation in terms of the Rydberg function,

$$
E_{\mathrm{i}}^{*}\left(a^{*}\right)=-\left(1+a^{*}\right) \exp \left(-a^{*}\right),
$$

to scale interaction energies of flat interfaces between pairs of metals. Here $E_{\mathrm{i}}^{*}=E_{\mathrm{i}} / E_{\mathrm{b}}$ and $a^{*}=\left(z-z_{\mathrm{e}}\right) / \lambda$, and $\lambda$ either is taken to be the Thomas-Fermi screening length or is considered as a fitting parameter. This scaling of the 

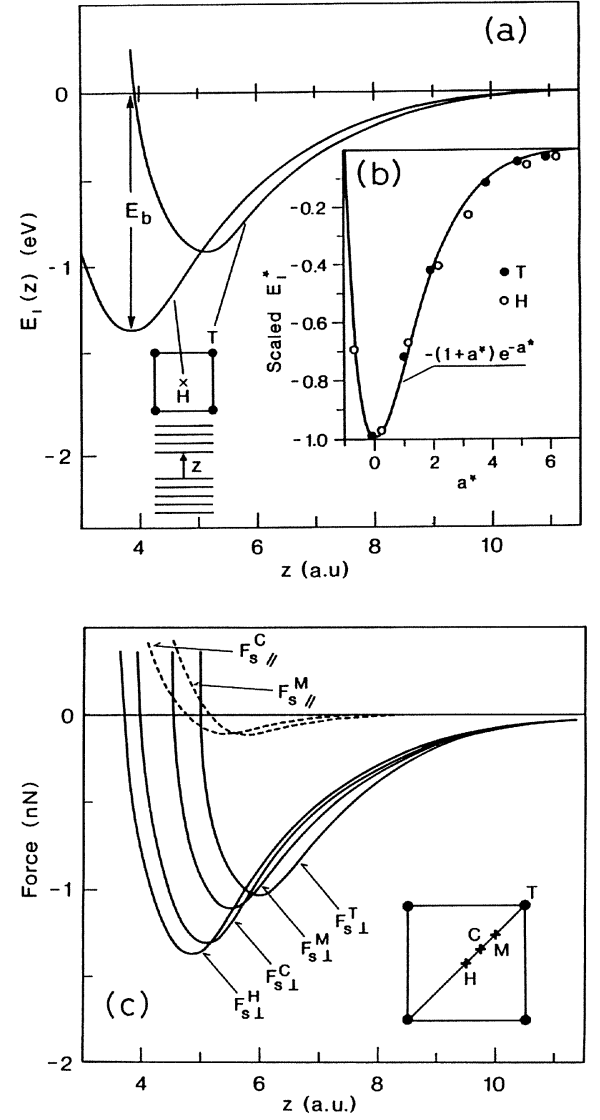

Fig. 8.3. (a) Interaction energy $E_{\mathrm{i}}$ versus separation $z$ between two rigid $\mathrm{Al}(001)$ slabs at the hollow $(\mathrm{H})$ and top $(\mathrm{T})$ sites. The $z$-axis is perpendicular to the (001)-plane. $E_{\mathrm{b}}$ is the minimum value corresponding to the binding energy. (b) Scaled energy $E_{\mathrm{i}}^{*}$ versus scaled separation $a^{*}$ according to the Rydberg function of Rose et al. [8.37a]. (c) Perpendicular $F_{\mathrm{s} \perp}$ and lateral $F_{\mathrm{s} \mid}$ short range forces (in nano Newtons) on the single-layer "tip slab" versus separation $z$. Forces are calculated for $\mathbf{H}-, \mathrm{C}-$, $\mathrm{M}$-, T-sites shown in the inset

adhesive energy has been exploited by Dürig et al. [8.9] to fit the variation of the force gradient with a separation in a combined atomic force and tunneling microscopy experiment. By merely taking $\lambda=1$ a.u. for Al slabs, the scaled interaction energies $E_{\mathrm{i}}^{*}$ in Fig. $8.3 \mathrm{~b}$ fit very well to the Rydberg function at both the $\mathrm{T}$ - and $\mathrm{H}$-site.

\subsection{Short-Range Forces}

The force on an individual atom $j$ of the tip can be calculated either from the derivative of the calculated interaction energy, $F_{\mathrm{s}}(r)=-\nabla_{j} E_{\mathrm{i}}(\boldsymbol{r})$, or more conveniently from $\left\langle\nabla_{j} H_{\mathrm{LDA}}\right\rangle$ [8.38]. Indeed, once self-consistency has been achieved, changes in the wave function due to the displacement of nuclei do not contribute to the force, since the eigenfunctions are obtained variationally [8.39]. As a consequence, the force $\boldsymbol{F}_{\mathrm{s}}$ can be expressed as the sum of the 
ion-electron attraction (in which the electron density is calculated from the self-consistent wave functions),

$$
\sim 2 \int\left[\varrho_{\mathrm{S}}(\boldsymbol{r})+\Delta \varrho(\boldsymbol{r})\right]\left[\frac{\partial}{\partial \tau_{j}}\left(\frac{Z_{j}}{\left|\tau_{j}-\boldsymbol{r}\right|}\right)\right] \mathrm{d} \boldsymbol{r},
$$

and the ion-ion repulsion

$$
-\sum_{\mathrm{S}} \frac{\partial}{\partial \tau_{j}}\left(\frac{Z_{j} Z_{\mathbf{s}}}{\left|\tau_{j}-\tau_{\mathrm{s}}\right|}\right)
$$

which essentially compensate each other almost completely at large separations. In the above equations, $\tau_{\mathrm{S}}$ is the position vector of a sample atom, $\varrho_{\mathrm{S}}(\boldsymbol{r})$ is the charge density of the bare sample, $\Delta \varrho(r)$ is the modification of charge density due to the tip-sample interaction. In Fig. 8.3c, the variation of the perpendicular and parallel components of the total short-range force $\boldsymbol{F}_{\mathrm{s}}$ is shown for different lateral positions relative to the sample [8.33]. In the force calculations the sample consists of 5 layers $\mathrm{Al}(001)$ slab, and the tip is represented by a single $\mathrm{Al}(001)$ layer. The strongest attraction occurs at the smallest $z_{\mathrm{e}}$ at the $\mathrm{H}$-site. As the tip slab is moved from the $\mathrm{H}$-, towards the T-site the minimum of the perpendicular force curves gradually shifts to larger $z$ and concomitantly the strength of the attraction decreases. The calculated force curves indicate a corrugation $\Delta z \simeq 1.2$ a.u. at a constant loading force $F_{\mathrm{s} \perp}$ in the range of $\pm 1 \mathrm{nN} /$ atom.

The force variation depicted in Fig. $8.3 \mathrm{c}$ is also common to the outermost tip atom. At small $z$ the ion-ion repulsion in (8.5) is much larger than the ion-electron attraction in (8.4) and yields a repulsive force. As $z$ increases, $F_{\mathrm{s} \perp}$ changes sign where $E_{\mathrm{i}}(z)$ for a single atom attains its minimum value leading to a net attraction. This is mainly caused by the bonding charge density $\Delta \varrho(r)$ in (8.4) which is accumulated between the tip and sample. In the strong repulsive regime at small $z$, ion-ion repulsion considerably exceeds the magnitude of the ion-electron part at the T-site and is also larger than the ion-ion repulsion at the H-site. Consequently, $F_{\perp}^{\mathrm{T}}>F_{\perp}^{\mathrm{H}}$. As shown in Fig. 8.3c ion-ion repulsion continues to determine the force variation in the range $z \lesssim 6$ a.u. For $z \gtrsim 6$ a.u. the attractive force at the $\mathrm{T}$-site exceeds that at the $\mathrm{H}$-site, since attraction due to the change in the charge density $\Delta \varrho(r)$ dominates the ion-ion repulsion which becomes weaker at large $z$. Accordingly, the outermost tip atom images the ions of the sample surface at the repulsive and at the strongly attractive force range. At the weak attractive force range the charge density of the sample is imaged. This picture of force variation at the apex atom becomes complicated if the short- and long-range forces at the back of the apex are included. For example, the outermost atom may feel strong repulsion while the total (measured) tip force is still attractive.

Short-range lateral forces which produce energy losses through the energy transfer to shear modes, are the primary cause of friction. Recent measurements 
by Mate et al. [8.17] on the lateral forces as a function of perpendicular loading force and scan velocity have revealed important microscopic features of the friction phenomena. In particular, the dissipation of energy during the stick-slip motion requires further study on the atomic scale. Zhong and Tomanek [8.40] have provided a theoretical estimate of the friction coefficient $\mu$ from the atomic scale calculations on the slip of a commensurate Pd monolayer against graphite. They assumed that, in the limit of slow tracking velocity, the gain of conservative energy in going from the $\mathrm{H}$-site to the $\mathrm{T}$-site is fully dissipated in the opposite sequence. This, however, yields a rather unrealistic variation of energy dissipation with tip position. The experimental data [8.17] showing an average nonconservative force superposed on a conservative force, modulated with the lateral periodicity of the sample seems to be at variance with the theoretical results [8.40].

The thorough analysis of friction on the microscopic scale requires a detailed analysis of the energy dissipation. The scope of our present discussion is, however, limited to the analysis of lateral forces which are precursor to the friction. In Fig. 8.3c the variation of the lateral force $F_{\mathrm{s}||}(z)$, which is calculated self-consistently for the two $\mathrm{Al}(001)$ slabs is presented. They are directed along the diagonal HT in the surface unit cell, and by symmetry they vanish at the H-, and T-sites. For the present system they are one order of magnitude smaller than the perpendicular forces in a wide range of $z$, since attractive contributions from all neighboring sample atoms tend to add up in $\boldsymbol{F}_{\mathrm{s} \perp}$, but they tend to cancel out in $F_{\mathrm{s}||}$. Furthermore, $F_{\mathrm{s}||}(z)$ is not proportional to $F_{\mathrm{s} \perp}(z)$, although it exhibits a similar variation. For $z \gtrsim z_{\mathrm{e}}, F_{\mathrm{s} \|}$ is directed towards $\mathrm{T}$, but for $z \lesssim z_{\mathrm{e}}$ its direction is reversed. Nevertheless at $z \simeq z_{\mathrm{e}}, F_{\mathrm{s} \|}$ is finite, even though $F_{\mathrm{s} \perp} \sim 0$. This implies that the interaction-energy surface, $E_{\mathrm{i}}(r)$ does not have an absolute minimum between $\mathrm{M}$ - and $\mathrm{C}$-points.

\subsection{Deformations}

Forces acting on the tip induce deformations (due to the reversible or irreversible change of atomic structure). As body forces the VdW interactions by themselves do not cause any deformation, but their resultant is added to the short-range force. At large $z$ the tip force (and its reaction to the sample) is small and leads to small reversible deformations. In the same range the energy can further be lowered due to the avalanche in adhesion [8.41]. We first discuss the elastic (reversible) deformations.

The elastic deformation of the tip (and also of the sample in the vicinity of the tip) occurs in the weak attractive force range; its effect on the images was shown to be marginal [8.15]. Assume that the current $I$ is preset at the $\mathrm{H}$-site at a given $z_{I}$ which is larger than the separation corresponding to maximum attraction at the T-site. According to Fig. $8.3 \mathrm{c}$ the force on the outermost tip atom is $F_{\perp}^{\mathrm{H}}\left(z=z_{I}\right)$, and tunneling still occurs in that range, so that $I$ sensitively depends 
on the location of the tip. While scanning from the $\mathrm{H}$-site to the T-site the tip retracts by an amount $\Delta z_{I}$ corresponding to the corrugation of " $\varrho_{\mathrm{S}}\left(r, E_{\mathrm{F}}\right)$ ". This causes the attractive force either to decrease or to increase depending upon the variation of $F_{\mathrm{s} \perp}(z)$ in the weak attractive range. In the former case, which occurs when the $F_{\perp}^{\mathrm{H}}(z)$ curve lies below the $F_{\perp}^{\mathrm{T}}(z)$ curve and hence $\left|F_{\perp}^{\mathrm{T}}\left(z_{I}+\Delta z_{I}\right)\right|<\left|F_{\perp}^{\mathrm{H}}\left(z_{I}\right)\right|$, the outermost tip atom relaxes inwards leading to a greater separation at the T-site. The current $I$ would decrease, but the STM feedback control will move the tip towards the sample to maintain the preset current. As a result the recorded perpendicular motion of the tip holder is therefore smaller than $\Delta z_{I}$ in the absence of deformation. Earlier, based on the SCF-pseudopotential calculations it was found that the corrugation at constant $I$ is slightly reduced by elastic deformations [8.15]. In the latter case, where the $F_{\perp}^{\mathrm{H}}(z)$ curve lies above the $F_{\perp}^{\mathrm{T}}(z)$ curve and hence $\left|F_{\perp}^{\mathrm{T}}\left(z_{I}+\Delta z_{I}\right)\right|>\left|F_{\perp}^{\mathrm{H}}\left(z_{I}\right)\right|$, the apparent corrugation can be slightly enhanced if the real corrugation is smaller than the force corrugation at constant force. Nevertheless, the effect of elastic deformation on the STM corrugation is marginal.

Smith et al. [8.41] proposed an interesting effect which may be relevant to the deformation of the tip at small $z$. Based on the numerical calculations they showed that outermost atomic layers avalanche together when the separation of two electrodes falls below a critical distance, even if it is much larger than the equilibrium interfacial separation. An avalanche can occur regardless of the stiffness of external supports. Normally, the interaction energy would follow the curve in Fig. 8.3a, if the interlayer spacings were kept fixed. Smith et al. [8.41] showed that the interaction energy can be further lowered if the outermost layers are allowed to relax. The larger is the gain of energy the more surface layers are involved in the relaxation. The energy of the system is lowered since the energy gained by the attraction (or bond formation) of the surface layers of two electrodes exceeds the strain energy due to the increased surface and subsurface interlayer distances.

In the range of separation, yielding increasing attraction near $z=z_{\mathrm{e}}$ where $\partial F_{\mathrm{s} \perp} / \partial z>0$ several irreversible even hysteric effects take place, eventually leading to a plastic deformation [8.25, 26, 41, 42]. Extensive computer simulations using an empirical potential have described features of various atomistic mechanisms [8.26, 41, 42, 43] in this range of separation. Important aspects revealed from these computer simulations are surface melting, nanoindentation, formation of a connective neck, wetting mechanism and hysteresis of the retracting tip. Normally, the mechanical contact is expected to occur when the separation between the outermost tip and sample atoms is near $z_{\mathrm{e}}$. At this point the force on the outermost tip atom is zero but the total tip force is still attractive. Advancing the tip further results in a nanoindentation on the sample surface. Nanoindentation gives rise to a local plastic deformation involving massive atomic displacement, and perhaps the mixing of tip and sample atoms. When retracting, the tip does not trace the same force and energy curve it does when advancing. This is associated with the hysteresis. In retracting the tip, a connective neck forms first between the two electrodes. Finally, even if the tip 
is completely separated from the sample, atoms of one electrode may wet the other. Which atoms form the connective neck, and also which electrode is wetted, depend mainly on the relative binding energies of electrode atoms (i.e., tip atom on the tip $E_{\mathrm{tt}}$, sample atom on the sample $E_{\mathrm{Ss}}$, tip atoms on the sample $E_{\mathrm{ts}}$ and sample atoms on the tip $E_{\mathrm{St}}$. In general, the tip atom wets the sample if $\left|E_{\mathrm{ts}}\right|>\left|E_{\mathrm{tt}}\right|$ and/or the cohesive energy of the sample is stronger than that of the tip.

\subsection{Atom Transfer}

In the previous section we described the irreversible deformations which are due to the close proximity of the tip at the mechanical contact. Here we present microscopic aspects of the atom transfer which may occur already before mechanical contact, but may lead to an irreversible modification of the atomic structure [8.44-46]. Earlier, Gomer [8.47] discussed possible mechanisms of atom transfer in STM using schematic variation of interaction energy near the tip and sample. Pointing out atom tunneling, thermally activated desorption and field desorption, he concluded that thermal desorption could be responsible for the sudden transfer of an atom to or from the tip.

The atom at the apex of a sharp tip has a smaller coordination and thus weaker binding compared to that in the bulk. As the tip approaches the sample, the apex atom is attracted towards the sample. To visualize what can happen we can consider a single tip atom between a flat tip and a flat sample. Then, two interaction energy curves like those in Fig. 8.3a can be visualized as being attached to each electrode in opposite directions. The resulting $E_{\mathrm{i}}(z)$ curve rises to very large values in the repulsive range at very small separations from the electrodes, but has two minima separated by an activation barrier $Q_{\mathrm{b}}$ if $s \gtrsim 2 z_{\mathrm{e}}(s$ being the distance between two flat electrodes). Figure 8.4 illustrates the evolution of the exact interaction energy versus the position $z$ of an $\mathrm{Al}$ atom between two $\mathrm{Al}(001)$ slabs calculated as a function of their separation $s$. The curve has a reflection symmetry at the center since the $\mathrm{Al}$ atom is taken to be located between the $\mathrm{H}$-sites of both surfaces. When the atom is far away from one electrode, the interaction energy with the other becomes identical to its interaction energy curve with a single electrode. As $s$ decreases, the two surfaces interact and hence the actual energy is expected to deviate from that obtained by superposing two $E_{\mathrm{i}}(z)$ curves. Therefore calculations like that presented in Fig. 8.4 become important when $s$ is small. As expected, $Q_{\mathrm{b}}$ decreases with decreasing $s$ and hence the rate of the apex atom flicking back and forth to exchange its position at a given temperature increases. The rate of thermal desorption is given by [8.47]

$$
v \exp \left[-Q_{\mathrm{b}}(s) / k_{\mathrm{B}} T\right]
$$

where $v$ is the attempt frequency of $10^{12} \mathrm{~s}^{-1}$. In the course of the approach to the 


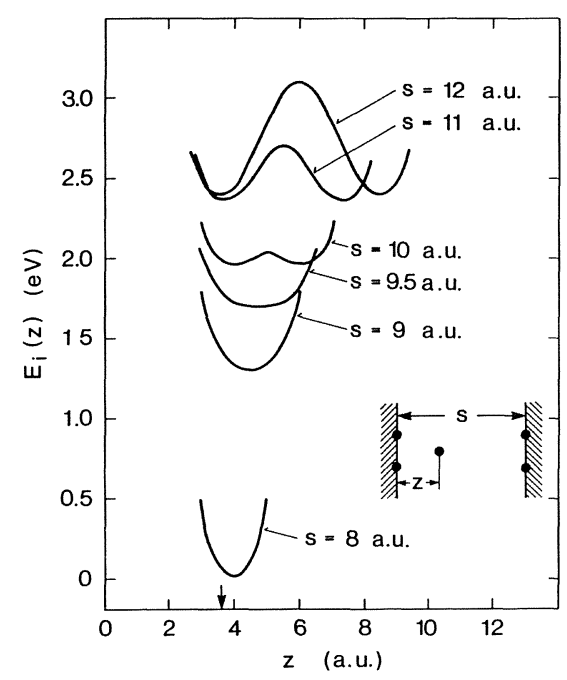

Fig. 8.4. Interaction energy of a single $\mathrm{Al}$ atom between two $\mathrm{Al}(001)$ slabs versus its distance, $z$ from the left electrode as described in the inset. The single $\mathrm{Al}$ atom faces to the $\mathrm{H}$-site of both $\mathrm{Al}(001)$ slabs

sample, the height of the apex atom from the rest of the tip gradually increases with increasing sample interaction. Eventually, the barrier collapses upon further advancing of the tip. At the instant $Q_{\mathrm{b}} \approx 0$ the distance of the apex atom both from the sample surface and from the rest of the tip is larger than that corresponding to the equilibrium distances achieved with only one electrode. This behavior is reminiscent of the avalanche effect discussed in the previous section [8.41] leading to a discontinuous drop in the interaction energy. Here the tip (except the outermost atom) and sample are held rigid, so that the interlayer separations are kept fixed. The extend of the curves in Fig. 8.4 would change if the fully relaxed electrodes were taken into account.

In the phenomenon illustrated in Fig. 8.4 the strain energy generated by advancing the outermost atom from the rest of the tip is balanced by the increased attractive interaction from the sample, since the atom gets closer to the sample. In the end, the binding is achieved with a considerably larger binding energy occurring at a relatively large separation as compared to the binding with only one electrode. It means that the outermost atom is stabilized between two electrodes. This phenomenon may have several important implications in STM: (i) The probability of atom transfer towards the deeper minimum is higher for a tip-sample system yielding an asymmetric interaction energy curve. This implies that atoms of one electrode can be transferred to the one which provides stronger cohesion even before the barrier $Q_{\mathrm{b}}$ is collapsed. The asymmetry of the interaction energy curve can arise due to the fact that tip and sample are made of different materials. The tip is usually made from a hard material like W or Ir. Even if the tip and sample were made from the same material, their shapes and hence their coordination numbers would be different. The tip going away from the $\mathrm{H}$-site will experience a shallower minimum. Moreover, there are special places on the sample surface (like step edges and 
kinks) that may provide a deeper minimum. The atom transfer as described above is expected to be crucial for the wetting. One electrode having a much deeper minimum will be unaffected by the position of the tip and will continue to attract atoms from the other side. (ii) As in field desorption, the atom transfer to one side can be enhanced. An external field of $\sim \mathrm{V} / \AA$ can do this, since it can penetrate both the tip and the sample surface [8.48]. As a result of a controlled lateral and perpendicular motion of the tip under an appropriate electric field, the atoms can be relocated to the desired positions [8.44-46]. It is also argued that atoms are transferred by electromigration. (iii) When the tip and sample are brought together in mechanical contact, the size of the single atom contact may not be sufficient to open the lowest ballistic channel with a quantum conductance $2 e^{2} / h$, even if the atom between the electrodes may have resonance states near the Fermi level. This situation is similar to a double-barrier quantum well and gives rise to a jump in the conduction even if the first channel is not opened. The same situation was found earlier in impurity scattering in a 1D mesoscopic channel [8.49-52]. Of course, the resonance condition varies with geometrical configuration (or with s) as the tip scans above the sample surface.

\subsection{Tip-Induced Modifications of Electronic Structure}

In the independent electrode limit corresponding to large $z$, the assumption that the tip as well as the sample states are unperturbed is justifiable. However, as the tip approaches the sample surface, the overlap of the tip and sample wave functions increases and a significant electronic interaction sets in. To understand such effects let us first consider unperturbed sample and tip wave functions $\Psi_{\mathrm{S}}$ and $\Psi_{\mathrm{t}}$ with energies

$$
\varepsilon_{\mathrm{S}}=\left\langle\Psi_{\mathrm{S}}\left|H_{\mathrm{S}}\right| \Psi_{\mathrm{S}}\right\rangle, \quad \varepsilon_{\mathrm{t}}=\left\langle\Psi_{\mathrm{t}}\left|H_{\mathrm{t}}\right| \Psi_{\mathrm{t}}\right\rangle,
$$

respectively. To simplify the picture we also assume that $\left\langle\Psi_{\mathrm{S}} \mid \Psi_{\mathrm{t}}\right\rangle=0$. For the interacting tip-sample system the total Hamiltonian $H_{\mathrm{S}+\mathrm{t}}$ differs from the sum of $H_{\mathrm{S}}$ and $H_{\mathrm{t}}$. Then, in first-order perturbation theory the hopping energy at a given $z$

$$
U_{\mathrm{S}, \mathrm{t}}(z)=\left\langle\Psi_{\mathrm{S}}\left|H_{\mathrm{S}+\mathrm{t}}\right| \Psi_{\mathrm{t}}\right\rangle
$$

measures the interaction between tip and sample. When $U_{\mathrm{S}, \mathrm{t}}(z)$ is small the energies of the independent electrode states shift slightly without a significant mixing. In general the smaller $z$ and $\left|\varepsilon_{\mathrm{t}}-\varepsilon_{\mathrm{S}}\right|$, the larger is $U_{\mathrm{S}, \mathrm{t}}$. If no other states engage in the interaction, these interacting electrode states form bonding, $\left(\Psi_{+}=c_{+} \psi_{\mathrm{s}}+c_{-} \psi_{\mathrm{t}}\right)$ and antibonding $\left(\Psi_{-}=c_{-} \Psi_{\mathrm{S}}-c_{+} \Psi_{\mathrm{t}}\right)$ combinations of the unperturbed tip and sample wave functions. In terms of $\xi=\left[4 U_{\mathrm{S}, \mathrm{t}}^{2}+\left(\varepsilon_{\mathrm{t}}-\varepsilon_{\mathrm{S}}\right)^{2}\right]^{1 / 2}$ the coefficients are given by

$$
c_{+}=\left[1 / 2+\left(\varepsilon_{\mathrm{t}}-\varepsilon_{\mathrm{S}}\right) / 2 \xi\right]^{1 / 2}, \quad c_{-}=\left[1 / 2-\left(\varepsilon_{\mathrm{t}}-\varepsilon_{\mathrm{S}}\right) / 2 \xi\right]^{1 / 2} .
$$


The energy of the bonding $\left(\Psi_{+}\right)$and antibonding $\left(\Psi_{-}\right)$states are expressed as

$$
\varepsilon_{ \pm}=\left\langle\Psi_{ \pm}\left|H_{\mathrm{S}+\mathrm{t}}\right| \Psi_{ \pm}\right\rangle=\left(\varepsilon_{\mathrm{t}}+\varepsilon_{\mathrm{S}}\right) / 2 \mp \xi / 2 .
$$

The admixture $1-c_{+}^{2}$ is a measure of the deviation from the independent electrode approximation. If $\varepsilon_{\mathrm{t}} \neq \varepsilon_{\mathrm{S}}$, mixing due to $U_{\mathrm{s}, \mathrm{t}} \neq 0$ results in a transfer of charge. Transfer of charge can occur even if $z$ is large and STM operates in the conventional tunneling regime. The charging effect has interesting implications. For example, an additional Coulomb attraction between tip and sample is induced as a result of the charging effect.

Earlier, Tekmain and Ciraci [8.12] pointed out that, owing to the local character of the perturbation, $\Psi$ becomes increasingly localized as $z$ is increased. For an actual tip-sample system the corresponding states become continuous, resulting in the density of $\varepsilon_{\mathrm{S}}$ and $\varepsilon_{\mathrm{t}}$. In this situation, $\Psi_{ \pm}$become resonance states. An instructive way to look at these states is to think of the tip as creating a local perturbation in the potential near the surface of the sample. Just like a surface defect [8.53], this perturbation can lead to localized states or resonances with enhanced amplitude in the vicinity of the tip. This can give rise to an anomalous $z$-dependence of STM images. One important difference is that the perturbation is dragged along as the tip is scanned over the sample. The observed modulation in $z$ (at constant tunneling current $I$ ) or in $I$ itself (at constant mean current) therefore reflects in part changes in electronic structure due to the varying local environment of the tip. Earlier, the observed anomalous corrugation on the nominally flat (111) surfaces of simple and noble metals with the periodicity of the surface unit cell were attributed to the tip-induced states [8.15] and hence to the modifications of the width of the barrier derived thereof [8.54]. Much more recently, Doyen et al. [8.55] also used tip-induced resonance states [8.12] to explain the anomalous STM corrugation observed on the $\mathrm{Al}(111)$ surface.

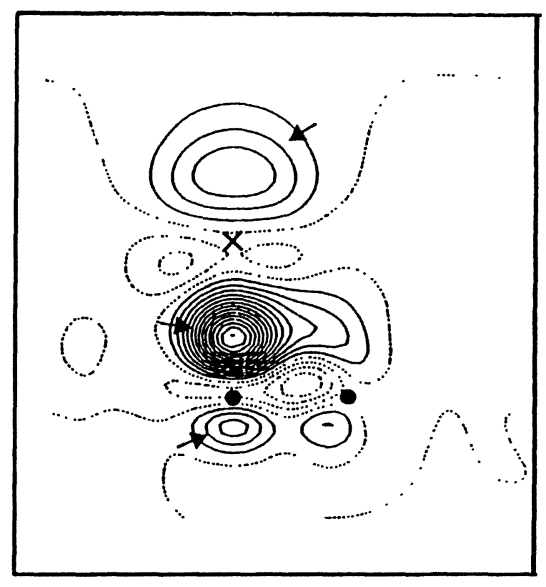

Fig. 8.5. Difference of total charge densities for a single graphite layer and the Al tip consisting of a single atom at $z=3.8$ a.u.. Aluminium and carbon atoms are indicated by a cross and dots, respectively. Dotted contours correspond to the charge depletion. Contour spacings are $2 \times 10^{-3}$ electrons $/(\text { a.u. })^{3}$ 


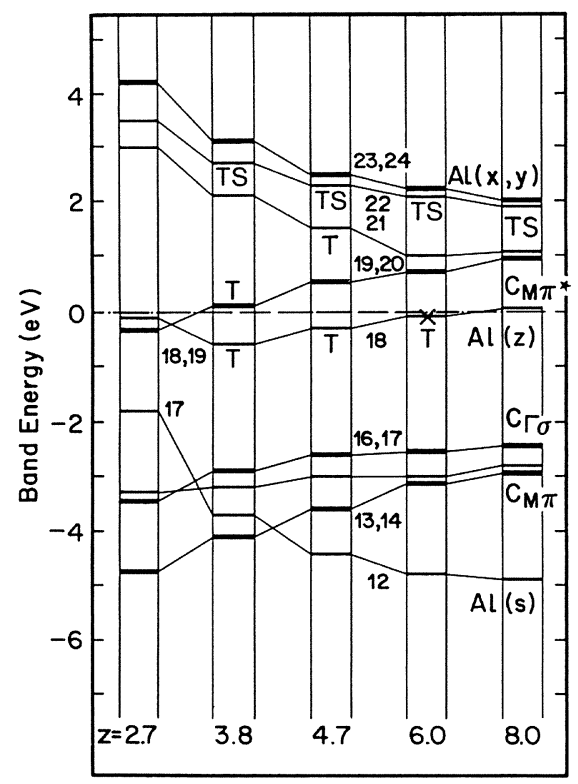

Fig. 8.6. The evolution of states of the graphite sample and the single $\mathrm{Al}$ atom tip as a function of separation for the H-site. $C_{M}$ states originate from the M-point of the graphite Brillouin zone. $\mathrm{Al}(x, y)$ and $\mathrm{T}$ (or TS) indicate $\mathrm{Al}\left(p_{x, y}\right)$ and tipinduced localized (or resonance) states, respectively

The bonding combination of $\Psi_{\mathrm{S}}$ and $\Psi_{\mathrm{t}}$ due to the tip sample interaction gives rise to charge accumulation between tip and sample surface. This can be seen in Fig. 8.5, in which the difference of total charge density [the total charge density of the combined tip-sample system minus the total charge densities of the bare electrodes, i.e. $\left.\Delta \varrho(r)=\varrho_{\mathrm{S}+\mathrm{t}}(\boldsymbol{r})-\varrho_{\mathrm{S}}(\boldsymbol{r})-\varrho_{\mathrm{t}}(\boldsymbol{r})\right]$, is shown. The energy difference, $\varepsilon_{+}-\varepsilon_{-}$increases with increasing $U_{\mathrm{S}, \mathrm{t}}$. As $z$ decreases, $\varepsilon_{+}$is lowered while $\varepsilon_{-}$raises. There might be a certain $\Psi_{+}$, which approaches to $E_{\mathrm{F}}$ at a critical $z$ and hence participates in tunneling. For example, in Fig. 8.6 it is shown that for graphite even the states at the M-point of the Brillouin zone, which are far away from the Fermi surface, can participate in tunneling at the $\mathrm{H}$-site as a result of a modification due to the close proximity of the tip. We note that in conventional tunneling the tip has to be advanced very close to the surface in order to get a significant current level from the $\mathrm{H}$-site of graphite.

\subsection{Calculation of Current at Small Separation}

According to the basic theory of STM the tunneling current decays exponentially, $I \propto \mathrm{e}^{-2 \kappa z}$, with $z$ and with the inverse decay length given by $\kappa=\sqrt{2 m \Phi_{\mathrm{B}} / \hbar}$ (or $\sqrt{\Phi_{\mathrm{B}}}$ in atomic units). Because of this exponential factor the tunneling current is extremely sensitive to $z$. Assuming that $\Phi_{\mathrm{B}}$ is independent of the lateral position of the tip, and also that the electronic states of the free sample are not disturbed by the tip, the variation of the measured tunneling 
current can be related to the variation of $\varrho_{\mathrm{S}}\left(\boldsymbol{r}_{0}, E_{\mathrm{F}}\right)$ of the unperturbed sample. However, it becomes clear from the discussion of the previous section that at small $z$ the electronic states are modified. The modifications have to be included in the calculation of the tunneling current. This is unfortunately very tedious [8.12].

In this section we discuss a method which starts with a realistic potential between electrodes and obtains the current $I$ (or conductance $G$ ) by evaluating the expectation value of the current operator with respect to the current carrying states calculated by using this potential $[8.24,29,51,54]$. Since the transfer Hamiltonian method is not used, we don't need the electronic states of bare electrodes and any induced modifications of these states explicitly. Nevertheless, these modifications are indigenous to our method since the changes in the electronic states and potential are interrelated. To formulate the transport, the tip-sample system is modeled by using two jellium electrodes separated by a vacuum potential barrier. Clearly the vacuum potential depends on the separation (the distance between two jellium edges $z_{j}$ ). Note that the separation $z_{j}$ is related to $z$. That is $z=z_{j}+d_{\mathrm{S}}+d_{\mathrm{t}}$ with $d_{\mathrm{S}}$ and $d_{\mathrm{t}}$ being half of the interlayer distances in the tip and sample. SCF calculations on a sharp tip facing the metal-sample surface have predicted a potential which is approximately quadratic in the transverse $(x y)$ plane in the vacuum gap [8.15]. Assuming cylindrical symmetry we express the potential in the vacuum gap as

$$
V(z, \boldsymbol{r})=\phi\left(z, z^{\prime}\right)+\alpha\left(z, z^{\prime}\right) \eta^{2} \theta\left(z^{\prime}+d_{\mathbf{S}} / 2\right) \theta\left(z^{\prime}-z+d_{\mathrm{t}} / 2\right)
$$

which is three dimensional. For a given tip-sample separation $z, V(z, \boldsymbol{r})$ varies with the position vector $\boldsymbol{r}=\boldsymbol{r}\left(x, y, z^{\prime}\right)$. The $(x y)$-plane is perpendicular to the $z^{\prime}$-axis, and $\eta^{2}=x^{2}+y^{2}$. The maximum of $\phi\left(z, z^{\prime}\right)$ coincides with the saddle point of $V(z, r)$ (i.e. $\left.V_{\mathrm{sp}}\right)$, and the barrier height is $\Phi_{\mathrm{B}}=\max \left\{\phi\left(z, z^{\prime}\right)\right\}-E_{\mathrm{F}}$. A schematic description of the potential expressed by (8.11) is presented in Fig. 8.7. This form of the potential allows a separable solution of the Schrödinger equation. Subsequent to the collapse of the barrier, the radius of the orifice with $V(z, r)<E_{\mathrm{F}}$ is $\eta_{\mathrm{m}}=\left\{\left[E_{\mathrm{F}}-V_{\mathrm{sp}}(d)\right] / \alpha\right\}^{1 / 2}$

The current carrying states are the $3 \mathrm{D}$ plane waves in the electrodes and the quantized states in the constriction defined by (8.11). Since $\phi$ and $\alpha$ vary with $z^{\prime}$,

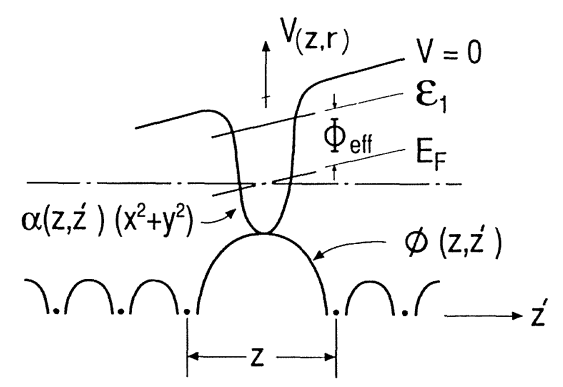

Fig. 8.7. A schematic representation of the potential expressed by (8.11) 
$V(z, r)$ is divided into segments. In each segment $\phi$ and $\alpha$ are assumed to be constant. Tekman and Ciraci [8.54] have expressed the current carrying solutions $\boldsymbol{\Psi}_{\mathbf{k}_{i}}$ corresponding to an incident wave $\boldsymbol{k}_{\boldsymbol{i}}$ deep in the tip electrode as

$$
\boldsymbol{\Psi}_{\boldsymbol{k}_{i}}\left(z, \eta, z^{\prime}\right)=\sum\left[A_{n k_{i}}\left(z, z^{\prime}\right) \mathrm{e}^{\mathrm{i} \gamma_{n}\left(z, z^{\prime}\right) z^{\prime}}+B_{n k_{i}}\left(z, z^{\prime}\right) \mathrm{e}^{\mathrm{i} \gamma_{n}\left(z, z^{\prime}\right) z^{\prime}}\right] \psi_{n}\left(z, \eta, z^{\prime}\right)
$$

where $\psi_{n}\left(z, \eta, z^{\prime}\right)$ is a $2 \mathrm{D}$ harmonic oscillator solution for a given $\alpha\left(z, z^{\prime}\right)$ with $j=j_{x}+j_{y}$ and $n=j+1\left(j_{x}, j_{y}=0,1,2, \ldots\right)$. The corresponding eigenenergy $\varepsilon_{n}\left(z, z^{\prime}\right)=n\left[2 \hbar^{2} \alpha\left(z, z^{\prime}\right) / m^{*}\right]^{1 / 2}$. The propagation constant is given by

$$
\gamma_{n}\left(z, z^{\prime}\right)=\left\{\frac{2 m^{*}}{\hbar^{2}}\left[E-\phi\left(z, z^{\prime}\right)-\varepsilon_{n}\left(z, z^{\prime}\right)\right]\right\}^{1 / 2} .
$$

The coefficients $A_{n k_{2}}$ and $B_{n k_{2}}$ in (8.12) are determined by using multiple boundary matching or transfer matrix methods $[8.51,56]$. The total tunneling conductance in terms of the matrices of coefficients $\gamma_{n}$ is obtained by integrating the expectation value of the current operator over the Fermi sphere. Details of derivative and final conductance expression in terms of matrices of coefficients and $\gamma_{n}$ are given in [8.51].

For a uniform and long orifice free from any scatterer (i.e., constant $\phi$ and constant $\alpha$ ) each state dipping below the Fermi level causes the conductance to jump by a quantum of conductance $2 e^{2} / h[8.29,57]$ and hence acts as a channel of conduction. Taking $E=E_{\mathrm{F}}$, the number of eigenstates $\varepsilon_{n}$ (including degeneracy $u_{n}$ ) which satisfy $\varepsilon_{n}+\Phi_{\mathrm{B}} \leq E_{\mathrm{F}}$ determine the number of independent conduction channels $N_{\mathrm{c}}=\Sigma_{n} u_{n}$. Then the total conductance of these independent channels becomes $G=\Sigma_{n} 2 e^{2} u_{n} / h$. The two terminal theories [8.58] predict that the conductance is given by $G=\left(2 e^{2} / h\right) \operatorname{Tr}\left(\boldsymbol{T}_{\mathrm{t}} \boldsymbol{T}_{\mathrm{t}}^{\dagger}\right)$ in terms of the matrix of transmission amplitudes $\mathbf{T}_{\mathrm{t}}$. In the absence of scatterers in a 1D conductor this expression becomes identical with the one obtained by using the independent channel arguments above. Recently, van Wees et al. [8.59] and Wharam et al. [8.60] achieved the measurement of the conductance $G$, through a narrow constriction between two reservoirs of a $2 \mathrm{D}$ electron gas in a high-mobility GaAs-GaAlAs heterostructure. The constriction they made by a split gate was sufficiently narrow that its width $w$ was comparable with the Fermi wave-length $\left(w \sim \lambda_{\mathrm{F}}\right)$, and also sufficiently short $\left(z<l_{\mathrm{e}}\right.$ electron mean free path) that electrons could move ballistically. The resulting conductance of the transport through this constriction was found to change with $w$ (or gate voltage) in quantized steps of approximately $2 e^{2} / h$. The sharp quantization is, however, smeared out or destroyed completely if $z$ is not long enough and if $\phi\left(z, z^{\prime}\right)$ is not uniform [8.51]. In this range the character of the conduction is ballistic. Whereas for $n$ with $\Phi_{\mathrm{B}}+\varepsilon_{n}>E_{\mathrm{F}}, \gamma_{n}$ in (8.13) becomes imaginary and yields evanescent wave solutions along the $z^{\prime}$-axis. The evanescent waves lead to the conduction by tunneling. The tunneling contribution is, however, negligible if the width of the barrier is large. According to the formalism discussed above, the conductance expression has a wide range of application and thus is appropriate for tunneling, ballistic and field emission [8.61]. 


\subsection{Constriction Effect}

The potential between the sample surface and the sharp tip displays the form of a narrow constriction, as expressed in (8.11). In the tunneling regime the maximum value of $V(z, r)$ (or saddle point value $V_{\mathrm{sp}}$ ) at $\eta=0$ may be higher than the energy of the highest occupied state. That is $\Phi_{\mathbf{B}}$ defined in the previous section is positive. Upon decreasing the separation $z$, the potential barrier $\Phi_{\mathrm{B}}$ decreases and eventually $V_{\mathrm{sp}}$ dips below $E_{\mathrm{F}}$ yielding a negative barrier. In a classical picture this situation can be viewed as a hole in the vacuum potential between electrodes leading to a ballistic transport. The contour plots of the potential for the $\mathrm{Al}$ tip and the $\mathrm{Al}(111)$ surface presented in Fig. 8.8 describe how a hole is formed in the potential barrier. To obtain the variation of the potential in the vacuum gap, the pyramidal tip was periodically repeated on the $\mathrm{Al}(111)$ slab resulting in a $(3 \times 3)$ tip array, and the charge density was calculated by using the SCF pseudopotential method. It is clearly seen that, near the plane bisecting $z, V_{\mathrm{sp}}>E_{\mathrm{F}}$ for $z=10$ a.u., whereas for $z=9$ a.u., $V_{\mathrm{sp}}$ dips into the Fermi level. In the transverse plane (or $x y$-plane)) the potential displays an approximately quadratic variation as in (8.11).

Clearly $V(z, r)$, having a quadratic variation in the $x y$-plane, is a constriction and imposes constraints in the motion of electrons in the $x$-and $y$-directions. As a result, the energy of the electron, which is confined to the radius of $\left[\left(E_{\mathrm{F}}-V_{\mathrm{sp}}\right) / \alpha\right]^{1 / 2}$, increases. For example, the lowest eigenstate of the electron confined to the 2D quadratic potential would occur at $\varepsilon_{1}\left(z, z^{\prime}\right)+$ $\left[2 \hbar^{2} \alpha\left(z, z^{\prime}\right) / m^{*}\right]^{1 / 2}$ above $V_{\mathrm{sp}}$. In the adiabatic approximation the effective
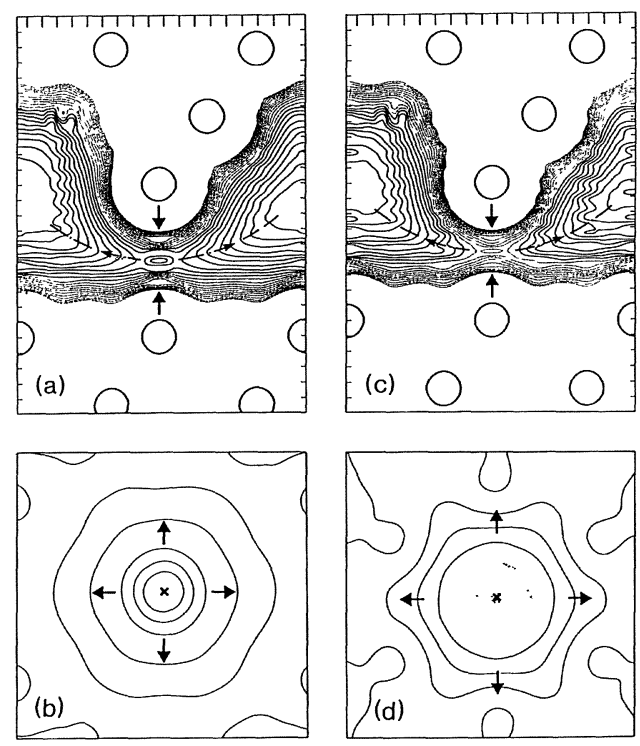

Fig. 8.8. Potential energy of a point contact which is created by a pyramidal $\mathrm{Al}$ tip on the $\mathrm{Al}(111)$ surface and calculated by the SCF pseudopotential method. (a) and (b) contour plots at $z=10$ a.u. for $(y z)$ and (xy)-planes, respectively. (c) and (d) are the same as for (a) and (b) but at $z=9$ a.u.. Solid and dotted curves correspond to $V(z, r)>E_{\mathrm{F}}$ and $V(z, r)<E_{\mathrm{F}}$, respectively. The potential increases in the directions indicated by arrows. The shaded area in (d) indicates the cross section of the orifice 
potential governing the $z$-dependence of the wave function without transverse nodes can be expressed as $V_{\text {eff }}=V\left(z, \eta=0, z^{\prime}\right)+\varepsilon_{1}\left(z, z^{\prime}\right)$. As long as $\Phi_{\text {eff }}=V_{\text {eff }}\left(z_{\mathrm{sp}}\right)+\left[2 \hbar^{2} \alpha\left(z, z^{\prime}\right) / m^{*}\right]^{1 / 2}-E_{\mathrm{F}}>0$, electronic states contributing to $I$ at small voltages experience an effective barrier of maximum height $\Phi_{\text {eff }}$ and are therefore evanescent in the constriction. This is the constriction effect which becomes crucial at small $z$, where $\Phi_{\mathrm{B}}$ is either positive but very low, or negative. As a result of the constriction effect, electrons going from one electrode to the other experience an effective barrier even if $\Phi_{\mathrm{B}}<0$. Because of this effect the resistance increases.

\subsection{Transition from Tunneling to Ballistic Transport}

The gradual collapse of the barrier and its effect on the current have been illustrated experimentally by Gimzewski and Möller [8.20] in studies of the transition from tunneling to point contact between an Ir tip and an $\mathrm{Ag}$ sample. Their $\log I$ versus apparent tip-displacement, $z_{\mathrm{a}}$ plot at constant voltage, clearly shows three regimes in the operation of STM discussed at the beginning of this chapter. Initially, the current $I$ increases first exponentially with decreasing $z$ (or increasing $z_{\mathrm{a}}$ ). This implies a tunneling behavior. The departure from linearity is due to the variation of height and width of the barrier due to the tip-sample interaction. The discontinuity observed at small $z$ was attributed to the mechanical instability (or irreversible deformation) [8.25]. The recorded values of the conductance just after the discontinuity was only $\sim 0.8$ of the unit of quantum conductance $\left(2 e^{2} / h\right)$. Upon further approach of the tip, $I$ continues to increase and exhibits an oscillatory behavior. Other experiments with different conditions showed different behaviors. For example, in some experiments $\log I\left(z_{\mathrm{a}}\right)$ did not increase, but saturated before any irreversible deformation took place. In some cases, at the point of mechanical instability $\log I\left(z_{\mathrm{a}}\right)$ is even decreased.

Different behaviors in those experiments, especially the oscillatory behavior following the mechanical instability, attracted interest. Important questions to be answered were how a plateau can occur prior to the plastic deformation, and what is the origin of the observed oscillations. Initially, the oscillatory behavior following the mechanical instability in the $\log I\left(z_{\mathrm{a}}\right)$ curve was interpreted as the manifestation of the quantized conductance [8.21]. Lang [8.22] simulated the point contact realized in the experiment [8.20] by two jellium electrodes, one of them having a $\mathrm{Na}$ atom attached to the jellium edge and thus representing a single atom tip. He found that the conductance $G$ saturates at the value $\zeta 2 e^{2} / h$ and forms a plateau when $z$ is in the range of the distance from the Na core to the positive background edge of the tip electrode. The value of $\zeta$ was only 0.4 for $\mathrm{Na}$, and was found to depend on the identity of the tip. Using the non-equilibrium Green's function method within the tight binding approximation Ferrer et al. [8.23] also found that $G$ reaches $\lesssim 2 e^{2} / h$ at the smallest $z$. Apparently, these studies [8.20-23] did not convey whether the transition from 
tunneling to ballistic regime already takes place at smallest $z$, or how a plateau can occur prior to the plastic deformation. Gimzewski and Möller [8.20] gave an estimate for the dimension of the contact area which lies in the range of $\lambda_{\mathrm{F}}$. If this is correct, the observed transport beyond the discontinuity has to be associated with the quantum ballistic transport [8.29].

Recent calculations $[8.24,51]$ of the current between an atomically sharp tip and a flat sample surface performed by using the method and the model potential described in Sect. 8.8 clarified some of the features in the $\log I\left(z_{\mathrm{a}}\right)$ curve. In order to link theory with experiment, $\alpha$ in (8.11) is obtained by using the diameter of contact given by experiment and by scaling those values calculated for the $\mathrm{Al}$ tip and $\mathrm{Al}$ sample [8.15] as a function of $z$. Furthermore, the electronic parameters of $\mathrm{Ag}$ are used for the jellium electrodes. The calculated $\log G$ (which has the same behavior as $\log I$ ) versus the tip displacement is illustrated in Fig. 8.9. In agreement with previous calculations $[8.22,23]$ the conductance associated with a uniform constriction set up by a single atom at the vertex of the tip has a value less than the quantum of conductance. Since the length of the constriction is finite and within the range of internuclear distance $a_{0}$ (i.e. the sum of atomic radii), this result implies that the energy of the first subband $\varepsilon_{1}$ is still above $E_{\mathrm{F}}$ (i.e. $\Phi_{\text {eff }}>0$ ) and, hence, the conductance is dominated by tunneling.

From the behavior of $\log I\left(z_{a}\right)$ in [8.20], it appears that ballistic transport sets in after the structural instability occurs at $z>a_{0}$. While $z$ cannot be smaller than $a_{0}$, by pushing the tip further, the contact area expands due to increasing plastic deformation followed by adhesion of nearby atoms. That is, while the apparent approach of the tip towards sample continues and hence $z_{\mathrm{a}}$ increases, the separation $z$ is stabilized at the value $\sim a_{0}$. This is marked by the vertical dash-dotted line in Fig. 8.9. The actual form and size of the contact after the point of mechanical instability is uncertain. Several parameters (such as the detailed atomic structure of the apex of the tip, the strengths of $E_{\mathrm{SS}}, E_{\mathrm{tt}}$, and $E_{\mathrm{St}}$ )

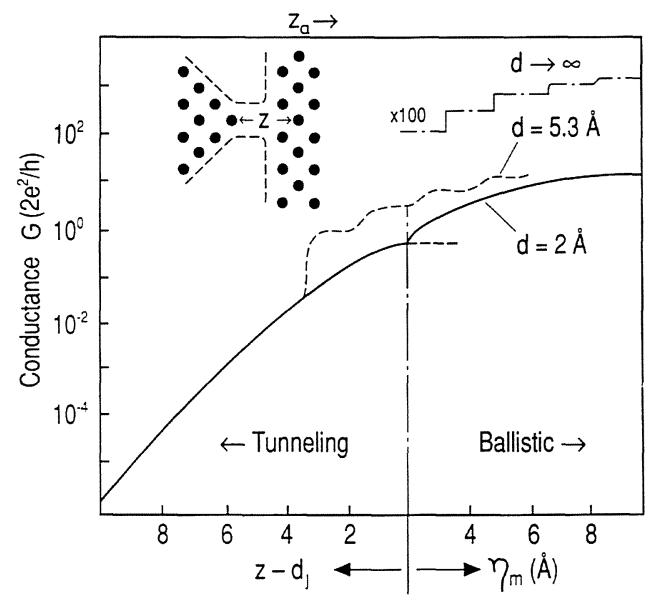

Fig. 8.9. Conductance $G$ versus separation $z$ (in $\AA$ ) or apparent displacement $z_{\mathrm{a}}$ (in arbitrary units) of an $\mathrm{Ag}$ tip above an $\mathrm{Ag}$ sample showing the transition from tunneling to ballistic conduction. The effective length of the constriction is $d \simeq z$. The term $\eta_{\mathrm{m}}$ is the radius of the constriction at $E_{\mathrm{F}}$. Quantum features are smeared out in the ballistic transport regime corresponding to $d=2 \AA$ (or 3.8 a.u.) shown by solid lines beyond the tunneling regime. Quantum features become apparent at the dashed line corresponding to $d=5.3 \AA$ $\left(\simeq \lambda_{F}\right)$. The dash-dotted line shows sharp quantum steps in the constriction of infinite length 
are expected to influence the contact. For example, the apex atom in the repulsive force range can be displaced in such a way that the aperture of the orifice is reduced, incidently causing $G$ to decrease temporarily in the course of the approach. Apart from this exceptional case, we assume that the radius of the contact increases upon approach of the tip. Then, the conductance is related to the radius $\eta_{\mathrm{m}}$ of the orifice, which increases normally with increased plastic deformation. As the aperture (or diameter) of the contact increases, subbands due to the quantization in the constriction dip sequentially into the Fermi level, causing $G$ to increase by the multiples of $2 e^{2} / h$ each time. As pointed out in Sect. 8.8, in spite of these expected jumps in $G$, the perfect quantization with a sharp step structure can only be observed if the length of the constriction is longer than $\lambda_{\mathrm{F}}$.

The variation of $G$ in the course of plastic deformation is examined by calculating the conductance as a function of $\eta_{\mathrm{m}}=\sqrt{E_{\mathrm{F}} / \alpha}$, for $\phi\left(z, z^{\prime}\right)=0$ at fixed effective length $d$ (which is approximately equal to $z$ ). Results are illustrated for $d=2,5.3 \AA$, and $\infty$. The first channel is opened at a radius as low as $\eta_{\mathrm{m}}=1.5 \AA$ followed by a rise of $\sim 2 e^{2} / h$ in $G$. The pronounced oscillations (or smeared-out step structure) of $\log G\left(\eta_{\mathrm{m}}\right)$ are apparent only for $d \simeq 5.3 \AA$, which is comparable with $\lambda_{\mathrm{F}}$. However, for $d \simeq a_{0}$, weak oscillations are washed out on the logarithmic scale. It is seen that the point contact between the tip and sample is not long enough to allow steps or pronounced oscillations. Therefore, the observed oscillations possibly originate from the irregular motion of the atoms as the tip is uniformly pushed towards the sample, causing irregular enlargement of the contact area. Also the atoms of a blunt tip may undergo sequential contacts, with each contact opening a new orifice and leading to abrupt changes in the current. Both cases can give rise to the variation of $\log I\left(z_{\mathrm{a}}\right)$ as observed experimentally.

The behavior of $G\left(z_{\mathrm{a}}\right)$ is further analyzed by using $\alpha(z)$ values corresponding to different tips. Depending on the shape and material properties of the tip, the form of $G\left(z_{\mathrm{a}}\right)$ may exhibit significant changes. For example, $G$ decreases, passing through a maximum $\left(\sim 2 e^{2} / h\right)$ if $\eta_{\mathrm{m}}$ is allowed to be less than the atomic radius of $\mathrm{Ag}$. On the other hand, if $\alpha$ is small, $G\left(z_{\mathrm{a}}\right)$ may reach a plateau before the point of discontinuity that results from the mechanical instability. This suggests that $\Phi_{\text {eff }}$ collapses prior to the hysteric deformation, but the value of $G$ at the plateau may be smaller than the quantum of conductance owing to scattering by the ions in the constriction. Certain contacts may have several subbands close to $E_{\mathrm{F}}$, each contributing to tunneling. In this case, plateaus do not occur, but $G\left(z_{\mathrm{a}}\right)$ increases almost linearly. For a flat tip (with several atoms at the apex) the contact area is large and, hence, $G$ rises above $2 e^{2} / h$ well before tip-induced plastic deformation sets in. It should be noted that all these arguments are based on the assumption that there is neither an oxide nor a flake between the tip and sample, as this would influence the variation of $G\left(z_{\mathrm{a}}\right)$ dramatically. The character of transport, and the variation of $G$ as a function of $z_{\mathrm{a}}$ (or $z$ ) are not generic, but are strongly tip and sample specific. The plateau can appear before the point of discontinuity only under certain conditions. 


\subsection{Tip Force and Conductivity}

In the discussion in Sects. 8.3, 4 it becomes clear that the short-range force becomes significant as long as the potential barrier between electrodes allow the wave functions to overlap. According to Bardeen's theory of tunneling [8.3] the conductance is also determined by the same overlap of wave functions at the Fermi level. Therefore, it is expected that the electrostatic force and conductance are interrelated. Earlier, the reversible modifications of electronic states prior to the contact, as well as correlations between force $F_{\mathrm{s} \perp}$ and barrier height $\Phi_{\mathrm{B}}$ were pointed out $[8.18,19]$. Furthermore, the shifts of the energy eigenvalues due to the proximity of the tip and hence the interaction energy of the tip-sample system were related to the hopping integral $U_{\mathrm{S}, \mathrm{t}}[8.19]$. Experimentally, Dürig et al. [8.9] drew attention to the correlation between force gradient and tunneling conductance $G$ in the course of the tip approach. More recently, following an idea of Herring [8.62], Chen introduced an expression which explicitly relates force with conductance [8.63]. First, in compliance with the discussion in Sect. 8.7, he obtains the interaction energy from the splitting of the coupled states through $\Sigma_{\mathrm{S}, \mathrm{t}} U_{\mathrm{S}, \mathrm{t}}(z)$. On the other hand, the hopping integral $U_{\mathrm{S}, \mathrm{t}}(z)$ itself is approximately equal to the tunneling matrix element $M_{\mathrm{S}, \mathrm{t}}(z)$ [8.64]. As a result, $E_{i}(z) \simeq \Sigma_{\mathrm{S}, \mathrm{t}} M_{\mathrm{S}, \mathrm{t}}(z)$, and hence the perpendicular component of the short-range force can be extracted from $\Sigma_{\mathrm{s}, \mathrm{t}} M_{\mathrm{s}, \mathrm{t}}(z)$, i.e. $F_{\mathrm{s} \perp} \simeq-\Sigma_{\mathrm{s}, \mathrm{t}} \partial M_{\mathrm{S}, \mathrm{t}} / \partial z$. This finally leads to $F_{\mathrm{s} \perp}=\xi \kappa \sqrt{G}$, since the tunneling conductance [8.4]

$$
G \propto \sum_{\mathrm{S}, \mathrm{t}}\left|M_{\mathrm{S}, \mathrm{t}}(z)\right|^{2} \delta\left(\varepsilon_{\mathrm{t}}-E_{\mathrm{F}}\right) \delta\left(\varepsilon_{\mathrm{S}}-E_{\mathrm{F}}\right)
$$

can be approximated in 1D tunneling by $G \propto \exp (-2 \kappa z)$ with $\kappa=\sqrt{\Phi_{\mathrm{B}}}$ in atomic units. It is clear that after all these simplifying approximations one cannot expect an exact expression among $F_{\mathrm{s} \perp}$, conductance $G$ and $\kappa$, as Chen [8.63] proposed. Nevertheless, his expression can be approximately valid as long as $\Phi_{\mathrm{B}}>0$, and becomes more accurate as $z$ increases beyond the point where $\Phi_{\mathrm{B}}$ collapses, since at large $z$ the interaction energy is better approximated by $\Sigma_{\mathrm{s}, \mathrm{t}} M_{\mathrm{S}, \mathrm{t}}(z)$. At this point, it is worth emphasizing that only the short-range force is related to the conductance. The overlap of wave functions is essential for tunneling conductance, but it is not invoked in the long-range force. Therefore, care must be taken in correlating measured tip force with conductance, since the experimentally determined force includes not only the short-range force, but also the long-range force depending upon the shape of the tip.

A possible relation between force and conductance can be sought by using calculated values. However, owing to the discretization in $\boldsymbol{k}$-space, the accurate calculation of the conductance is tedious with the above described SCF calculations. Here we compare calculated $F_{\mathrm{s} \perp}$ with $\kappa \exp (-\kappa z)$ (which corresponds to $\kappa \sqrt{G}$ in 1D tunneling). The difference between the maximum of the planar averaged potential at $z / 2$ and $E_{\mathrm{F}}$ is taken as the barrier height $\Phi_{\mathrm{B}}$ in the inverse decay length. Such a comparison indicates approximately a linear 
relation between the calculated short-range force $F_{\mathrm{s} \perp}$ and $\kappa \exp (-\kappa z)$. We note when correlating $F_{\mathrm{s} \perp}$ with $G$ that the image potential correction may be significant for large $z$. This correction is not included in the planar averaged potential. Moreover, neither the constriction effect due to a sharp tip, nor the 3D character of $G$ are taken into account. Despite the fact that several factors affecting the conductivity are not included, the linear relation between the calculated short-range force $F_{\mathrm{s} \perp}$ and $\kappa \exp (-\kappa z)$ corresponding to 1D tunneling is interesting.

In order to go beyond the approximation $G \propto \exp (-2 \kappa z)$ to calculate the conductance and to explore the relation between short-range force and conductance further, we use the jellium model following the method of Smith [8.36]. We first consider the electronic charge density of the semiinfinite left electrode, which is parameterized as $\varrho\left(z^{\prime}\right)=\varrho_{+}\left[1-\exp \left(-\beta z^{\prime}\right) / 2\right]$ for $z^{\prime}<0$ in the positive jellium and $\varrho\left(z^{\prime}\right)=\varrho_{+} \exp \left(-\beta z^{\prime}\right) / 2$ for $z^{\prime}>0$ in the vacuum region. Here $\varrho_{+}$is the positive jellium charge density corresponding to $\mathrm{Al}$, and $z^{\prime}=0$ marks the jellium edge. The charge density of the right electrode can be written with the same $\beta$ by replacing $-z^{\prime}$ by $z^{\prime}-z_{j}$. That the charge density is expressed in terms of a single exponent is, of course, an approximation, but is easily tractable. In the Hohenberg and Kohn theory [8.65] the ground state energy of a confined and interacting electron gas can be expressed as a functional of the electron number density $\varrho(r)$. Furthermore, this functional is minimized for the correct $\varrho(\boldsymbol{r})$. Accordingly, the exponent $\beta\left(z_{j}\right)$ of the charge density is determined by the minimization of the total energy (with nonlocal exchange and correlation potential Ref. 8.36) of the system consisting of two semiinfinite positive jellium at a given separation $z_{j}$. This way the modification of the electronic states due to electrode-electrode (or tip-sample) interaction can be taken into account to some extent [8.33].

Before we go further using simplified jellium approximation in calculating some transport properties, we compare its predictions with those obtained with the SCF pseudopotential method. For example, in the vacuum gap the planar averaged SCF potential is similar to that obtained by the jellium approximation, except that the barrier height in the former is slightly higher. The short-range force calculated by the jellium model compares well with the SCF pseudopotential method. For small separations the charge rearrangement cannot be described by a single $\beta\left(z_{j}\right)$. As a result, forces calculated by the jellium approximation become less attractive at small $z_{j}$. Also, for large $z_{j}$ one expects that the decaying tail of the charge density cannot be well represented by a single decay constant. As a matter of fact, at large separation even the SCF methods are unable to give an accurate representation of the tail of the charge density in terms of the plane-wave basis set. Nevertheless, the jellium method as described above is seen to provide a reasonable representation for the adhesion energy, forces and potential between two flat simple metal electrodes in the range $5 \lesssim z_{j} \lesssim 12$ a.u.

The transmission probability $T$ for electron tunneling through the vacuum barrier between two semiinfinite jellium slabs was calculated as a function of $z_{j}$ by using the transfer matrix method [8.56] and by using the WKB 
approximation [8.33]. For the sake of comparison $\exp \left(-2 \kappa z_{j}\right)$ and $\exp \left(-2 \kappa_{0} z_{j}\right)\left(\kappa_{0}\right.$ being the barrier height at infinite separation) are calculated. These quantities are relevant for tunneling through a vacuum barrier of constant height. The transmission probability $T$ calculated exactly becomes unity only when $z_{j}=0$ (i.e. when the two electrodes merge into one). For $z_{j}=0$, $\exp \left(-2 \kappa_{0} z_{j}\right)$ also becomes unity. However, the transmission probability obtained within the WKB approximation is unity when the barrier collapses for $z_{j} \lesssim 5$ a.u., which is also true for $\exp \left(-2 \kappa z_{j}\right)$. This shows that the WKB approximation fails to represent the transmission for $\Phi_{\mathrm{B}}<0$. Moreover, the transmission probability calculated by the WKB approximation overestimates the exact $T$, but becomes proportional to it at large separations as expected. In fact, for large separations all quantities described above become proportional since one has a rather flat and wide tunneling barrier.

Finally we explore how the (short-range) force and conductance are related in the calculations by using the jellium approximation [8.33]. To this end, the calculated force is compared with $\kappa \exp \left(-\kappa z_{j}\right)$ and $\kappa \sqrt{G}$ in Fig. 8.10. We note that in the jellium model the incident wave is a free electron state. Also the potential barrier is slightly underestimated as compared to the SCF results. A wide range of separation spanned in Fig. 8.10 cannot be described by the jellium aproximation, since a single $\beta\left(z_{j}\right)$ is not appropriate to represent the charge density for small and large separations. Moreover, for a very large separation $F_{\mathrm{s} \perp}$ is already too small. Hence only a limited range of the curves presented in Fig. 8.10 may be relevant for the present discussion. The force versus $\kappa \exp \left(-\kappa z_{j}\right)$ curve in Fig. 8.10a tends to indicate a power relation in the whole range. This power relation is not a simple one, perhaps approximately linear only in a limited range of $z_{j}$, where the attractive force is weak. Since $\exp \left(-2 \kappa z_{j}\right)$ is only an approximation to $1 \mathrm{D}$ conductance, force versus $\kappa \sqrt{G}$ in Fig. 8.10b (where $G$ is the 3D conductance calculated in the jellium approximation) is more relevant for our discussion. The latter curve also depicts a power relation, but no apparent linear relation throughout the whole range. As in the Fig. 8.10a the relationship can be viewed as linear only in a limited range of $z_{j}$, especially at the weak attractive force range. Our results provide an evidence
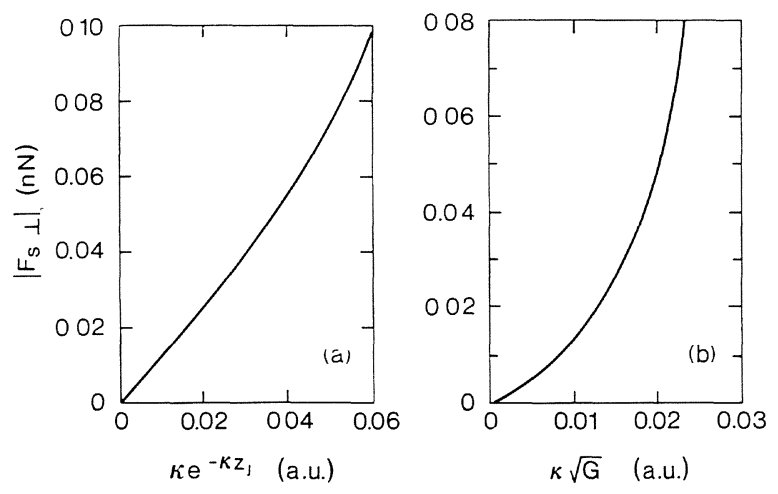

Fig. 8.10. Variation of force with $\kappa \exp \left(-\kappa z_{j}\right)(\mathbf{a}), \kappa \sqrt{G}$ (b); all quantities calculated by using the jellium approximation explained in the text 
that the short-range force can be related to some kind of transmission property of the barrier between two metal electrodes. Keeping in mind all the approximations involved in the present model, this should be perhaps a trend rather than an exact and universal relation. The overlap between sample and tip-wave functions, which determines both conductivity and short-range force, underlies such a relationship. Experimentally, deviations from any kind of universal expression are expected, depending on the conditions. For example, the structure of the tip enters as an essential parameter because of the constriction effect and also the contribution of the VdW force. Theoretical results obtained by different methods can also deviate due to the details of representing the vacuum potential.

\subsection{Summary}

In this chapter we discussed the interaction effects between the tip and sample in STM and SFM by varying their separation, and also by moving one over the other along certain symmetry directions. We presented results for the interaction energy, the perpendicular and lateral forces and the vacuum potential calculated earlier by using the SCF pseudopotential method. For the sake of comparison, the same physical quantities together with transmission and conductance between two semiinfinite slabs, which were calculated within the jellium approximation, were also presented. The important aspects of the chapter are summarized as follows: (i) the interaction energy and the short-range force are site-dependent, but both can be expressed by a Rydberg function in terms of the scaled energy and distance. (ii) The van der Waals forces are important at a small tip-sample distance if the tip is flat, or has a large cross section. However, the VdW force and its gradient have negligible effects if the tip is sharp. (iii) Calculated short-range force curves of simple metal electrodes indicate a corrugation of 1.2 a.u. for constant loading force varying in the range of $\pm 1 \mathrm{nN} /$ atom. (iv) The interaction energy curve having asymmetric double minima gives rise to the atom transfer. (v) At small separations tip-induced modifications of the electronic structure may give rise to resonance states dragged along by the tip. The tunneling current may be enhanced in the presence of these resonance states. (vi) A narrow constriction between tip and sample introduces an effective barrier; in its presence electrons tunnel, even if the potential barrier is collapsed. (vii) The ballistic transport between tip and sample is allowed if the diameter of the contact is comparable with the Fermi wavelength. However, sharp quantizations are smeared out. (viii) We presented some evidence that the tip force is related to the transmission property. This relation reflects, however, a nature of a trend rather than an exact and universal expression.

Acknowledgment. The author thanks Prof. E. Tekman for his valuable assistance and stimulating discussions during the preparation of the manuscript. This work was partially supported by the Joint Project Agreement between Bilkent University and IBM Zurich Research Laboratory. 


\section{References}

8.1 G. Binnig, H. Rohrer, Ch. Gerber, E. Weibel: Phys. Rev. Lett. 49, 57 (1982)

G. Binnig, H. Rohrer: I.B.M. J. Res. Dev. 30, 1 (1986)

G. Binnig, H. Rohrer: Rev. Mod. Phys. 59, 615 (1987)

8.2 G. Binnig, C.F. Quate, Ch. Gerber: Phys. Rev. Lett. 56, 930 (1986)

8.3 J. Bardeen, Phys. Rev. Lett. 6, 57 (1961)

8.4 J. Tersoff, D.R. Hamann: Phys. Rev. Lett. 50, 1998 (1983)

8.5 A. Baratoff: Physica B + C 127, 143 (1984)

8.6 A. Selloni, P. Carnevali, E. Tosatti, C.D. Chen: Phys. Rev. B 31, 2602 (1984); B 34, 7406 (1986)

8.7 L.D. Bell, M-.H. Hecht, W.J. Kaiser, L.C. Davis: Phys. Rev. Lett. 64, 2679 (1990)

8.8 H.W. Fink: IBM J. Res. Develop 30, 460 (1986); Phys. Scr. 38, 160 (1988)

H.W. Fink, W. Stocker, H. Schmidt, Phys. Rev. Lett. 65, 1204 (1990)

8.9 U. Dürig, O. Züger, D.W. Pohl: Phys. Rev. Lett. 65, 349 (1990)

U. Dürig, O. Züger, Vacuum, 41, 382 (1990)

8.10 J.M. Soler, A.M. Baro, N. Garcia, H. Rohrer: Phys. Rev. Lett. 57, 444 (1986)

8.11 S. Ciraci, I.P. Batra: Phys. Rev. B 36, 6194 (1987)

I.P. Batra, S. Ciraci: J. Vac. Sci. Technol. A 6, 313 (1988)

8.12 E. Tekman, S. Ciraci: Phys. Scr. 38, 486 (1988); Phys. Rev. B 40, 10286 (1989)

8.13 V.M. Hallmark, S. Chiang, J.F. Rabolt, J.D. Swalen, R.J. Wilson: Phys. Rev. Lett. 59, 2879 (1987)

8.14 J. Wintterlin, J. Wiechers, H. Brune, T. Gritsch, H. Höfer, R.J. Behm: Phys. Rev. Lett. 62, 59 (1989)

8.15 S. Ciraci, A. Baratoff, I.P. Batra: Phys. Rev. B 42, 7618 (1990)

8.16 G. Binnig, Ch. Gerber, E. Stoll, T.R. Albrecht, C.F. Quate: Europhys. Lett. 3, 1281 (1987)

T.R. Albrecht, C.F. Quate: J. Vac. Sci. Technol. A 6, 271 (1988)

R. Erlandsson, G.M. McClelland, C.M. Mate, S. Chiang: J. Vac. Sci. Technol. A 6, 266 (1988)

H. Heinzelmann, E. Meyer, P. Grütter, H.-R. Hidber, L. Rosenthaler, H.-J. Güntherodt: J. Vac: Sci. Technol. A 6, 275 (1988)

O. Marti, B. Drake, S. Gould, P.K. Hansma: J. Vac. Sci. Technol. A 6, 287 (1987)

8.17 C.M. Mate, G.M. McClelland, R. Erlandsson, S. Chiang: Phys. Rev. Lett. 59, 1942 (1987)

8.18 S. Ciraci: Tip-surface interactions, in Basic Concepts and Applications of Scanning Tunneling Microscopy and Related Techniques, ed. by H. Rohrer, N. Garcia, R.J. Behm (Kluwer Academic, Dordrecht 1990) p.119

8.19 S. Ciraci, A. Baratoff, I.P. Batra: Phys. Rev. B 41, 2763 (1990)

8.20 J.K. Gimzewski, R. Möller: Phys. Rev. B 36, 1284 (1987)

8.21 N. Garcia (unpublished)

8.22 N.D. Lang: Phys. Rev. B 36, 8173 (1987)

8.23 J. Ferrer, A. Martin-Rodero, F. Flores: Phys. Rev. B 38, 10113 (1988)

8.24 S. Ciraci, E. Tekman: Phys. Rev. B 40, 11969 (1989)

8.25 J.B. Pethica, W.C. Oliver: Phys. Scr. 19 A, 61 (1987)

J.B. Pethica, A.P. Sutton: J. Vac. Sci. Technol. A 6, 2490 (1988)

8.26 J.K. Gimzewski, R. Möller, D.W. Pohl, R.R. Schlitter, Surf. Sci. 189/190, 15 (1987)

U. Landman, W.D. Luedtke, N.A. Burnham, R.J. Colton: Science 248, 454 (1990)

U. Landman, W.D. Luedtke: J. Vac. Sci. Technol. B 9, 414 (1991)

8.27 S. Ciraci: Ultramicroscopy 42-44, 16 (1992)

8.28 N.D. Lang: Phys. Rev. Lett. 56, 1164 (1986); Phys. Rev. B 34, 5947 (1986)

8.29 G. Kirczenow: Solid State Commun. 68, 715 (1988)

L.I. Glazman, G.B. Lesovick, D.E. Khmelnitskii, R.I. Shekhter: Pis'ma Zh. Eksp. Teor. Fiz. 48, 238 (1988)

A. Szafer, A.D. Stone: Phys. Rev. Lett. 60, 300 (1989)

E. Tekman, S. Ciraci: Phys. Rev. B 39, 8772 (1989) and references therein

8.30 J.E. Inglesfield, E. Wikborg: J. Phys. F: Metal Phys. 5, 1475 (1975)

J.E. Inglesfield: J. Phys. F: Metal Phys. 6, 687 (1976)

J. Heinrich: Solid State Commun. 13, 1595 (1973) 
8.31 U. Hartmann: Phys. Rev. B 43, 2404 (1991)

8.32 F.O. Goodman, N. Garcia: Phys. Rev. B 43, 4728 (1991)

8.33 S. Ciraci, E. Tekman, A. Baratoff, I.P. Batra: Phys. Rev. B 46, 10411 (1992); S. Ciraci, E. Tekman, M. Gökcedag, A. Baratoff, I.P. Batra: Ultramicroscopy 42-44, 163 (1992)

8.34 E.M. Lifshitz: Soviet Physics, 2,73 (1956)

8.35 H.C. Hamaker: Physica 4, 1058 (1937)

8.36 J.R. Smith: Phys. Rev. B 181, 522 (1963)

J. Ferrante, J.R. Smith: Surf. Science. 38, 77 (1973)

A. Banerjea, J.R. Smith: Phys. Rev. B 37, 6632 (1988)

8.37 J.H. Rose, J. Ferrante, J.R. Smith: Phys. Rev. Lett. 47, 675 (1980)

J. Ferrante, J.R. Smith Phys. Rev. B 19, 3911 (1979)

8.38 H. Hellmann: Einführung in Quanten Theorie (Deutsch, Leipzig 1937)

R.P. Feynman: Phys. Rev. 56, 340 (1939)

8.39 J.C. Slater: J. Chem. Phys. 57, 2389 (1972)

8.40 W. Zhong, D. Tománek: Phys. Rev. Lett. 64, 3054 (1990) Europhys. Lett. 15, 887 (1991)

J.B. Sokoloff: Phys. Rev. Lett. 66 (1991) 965

8.41 J.R. Smith, G.B. Bozzolo, A. Banerjea, J. Ferrante: Phys. Rev. Lett. 63, 1269 (1989)

A.P. Sutton, J.B. Pethica: J. Phys. Cond. Matt. 2, 5317 (1990)

8.42 F. Abraham, I.P. Batra, S. Ciraci: Phys. Rev. Lett. 60, 1314 (1988)

F. Abraham, I.P. Batra: Surf. Sci. 209, L125 (1989)

8.43 E. Tosatti: "The Physics of Tip-surface Approaching: Speculations and open Issues" in Highlights of the Eighties and Future Prospects in Condensed Matter Physics ed. by L. Esaki (Plenum, New York 1990) p. 631

8.44 I.W. Lyo, P. Avouris: Science 245, 1369 (1989)

8.45 H.J. Mamin, P.H. Guethner, D. Rugar: Phys. Rev. Lett. 65, 2418 (1990)

8.46 D.M. Eigler, E.K. Schweizer: Nature, 344, 524 (1990)

D.M. Eigler, C.P. Lutz, W.E. Rudge: Nature, 352, 600 (1991)

8.47 R. Gomer: IBM J. Res. Develop. 30, 426 (1986)

8.48 H.J. Kreuzer: Surf. Sci. 246, 336 (1991)

8.49 C.G. Smith, M. Pepper, H. Ahmed, J.E.F. Frost, D.G. Hasko, D.C. Peacock, D.A. Ritchie, G.A.C. Jones: J. Phys. C 21, L893 (1988)

8.50 E. Tekman, S. Ciraci: Phys. Rev. B 40, 8559 (1990); Phys. Rev. B 42, 9098 (1990)

8.51 E. Tekman, S. Ciraci: Phys. Rev. B 43, 7145 (1991)

8.52 J. Faist, P. Gueret, H. Rothuizen: Phys. Rev. B 42, 3217 (1990)

8.53 H.A. Mizes, W.A. Harrison: J. Vac. Sci. Technol. A 6, 300 (1988)

8.54 E. Tekman, S. Ciraci: Phys. Rev. B 42, 1860 (1990)

8.55 G. Doyen, E. Koetter, J.P. Vigneron, M. Scheffler: App. Phys. A 51, 281 (1991)

8.56 P.E.O. Kane: In Tunneling Phenomena in Solids, ed. by E. Burstein, S. Lundqvist (Plenum, New York 1969)

8.57 R. Landauer, IBM J. Res. Dev. 1, 223 (1957); Z. Phys. B 68, 27 (1988); J. Phys. Cond. Matt. 1, 8099 (1989)

8.58 E.N. Economou, C.M. Soukoulis: Phys. Rev. Lett. 46, 618 (1981)

D.S. Fisher, P.A. Lee: Phys. Rev. B 23, 6851 (1981); G. Imry: In Directions in Condensed Matter Physics, ed. by G. Grinstein, G. Mazenko (World Scientific, Singapore 1986) p.101. For most general treatment see: M. Büttiker: Phys. Rev. Lett. 57, 1761 (1986); IBM J. Res. Develop. 32, 317 (1988)

8.59 B.J. van Wees, H. van Houten, C.W. Beenakker, J.G. Williams, L.P. Kouwenhowen, D. van der Marel, C.T. Foxon: Phys. Rev. Lett. 60, 848 (1988)

8.60 D.A. Wharam, T.J. Thornton, R. Newbury, M. Pepper, H. Ritchie, G.A.C. Jones: J. Phys. C 21, L209 (1988)

8.61 E. Tekman, S. Ciraci, A. Baratoff: Phys. Rev. B 42, 9221 (1990)

8.62 C. Herring: Rev. Mod. Phys. 34, 631 (1962)

8.63 C.J. Chen: J. Phys. Cond. Matt. 3, 1227 (1991)

8.64 F. Flores, A.M. Rodero, E.C. Goldberg, J.C. Duran: Nuovo Cimento 10, 303 (1988)

8.65 P. Hohenberg, W. Kohn: Phys. Rev. 136, B864 (1964) 